Int. J. Odontostomat., 6(2):139-143, 2012.

\title{
Estimación de la Estatura a Partir de las Dimensiones de la Dentición Temporal
}

\author{
Estimation of Stature from Dimensions of the Deciduous Dentition
}

Yasna Garrido*; Daniela Zavando** \& Iván Suazo Galdames ${ }^{* * *}$

GARRIDO, Y.; ZAVANDO, D. \& SUAZO, G. I. Estimación de la estatura a partir de las dimensiones de la dentición temporal. Int. J. Odontostomat., 6(2):139-143, 2012.

RESUMEN: La estimación de la estatura a partir de segmentos óseos o dentarios es un procedimiento complejo y con validez discutible, especialmente cuando se trata de restos humanos de subadultos. El objetivo de este estudio fue evaluar el método de Carrea para la determinación de la estatura a partir de las dimensiones de piezas temporales maxilares y en un segundo paso proponer un método matemático adecuado para la estimación de la estatura. Se determinó el arco y radio-cuerda maxilar en 42 pacientes de entre 36 y 84 meses y se aplicó el método de Carrea, comparando la estatura estimada con la real. Posteriormente se realizó una regresión lineal multivariada con la estatura como variable dependiente y el arco y radio-cuerda maxilar como variable independiente. El método de Carrea resultó negativo en todo los casos. Se determinó una ecuación de regresión que permitió la estimación correcta de la estatura en 57,5\% de los casos con $\pm 5 \mathrm{~cm}$ y en un $97 \%$ con $\pm 10 \mathrm{~cm}$, lo que supone una utilidad práctica para la estimación de la estatura que debe ser complementada con otros métodos.

PALABRAS CLAVE: estatura, indice de Carrea, odontología forense.

\section{INTRODUCCIÓN}

La determinación del sexo, la edad, la estatura y la raza del sujeto, esto constituye la fase inicial de identificación de restos óseos, estos antecedentes permiten construir un perfil biológico de un individuo, lo que constituye el primer paso para la identificación, cuando no es posible utilizar métodos convencionales de identificación basados en las huellas dactilares o en la identificación guiada por el reconocimiento de facciones y/o vestimentas (Cordeiro et al., 2009; Mantilla et al., 2009; Suazo et al., 2009b).

Este perfil se puede obtener a partir diferentes tipos de huesos, siendo los más utilizados: pelvis, cráneo, huesos largos (fémur, tibia, húmero, radio), vértebras cervicales, clavícula, esternón, costillas, calcáneo, metatarsianos, entre otros (Borborema et al., 2010; Suazo et al., 2008; Suazo et al., 2009a).

La estatura se considera exclusiva de la especie humana, ya que los animales no asumen una pos- tura erguida habitual fisiológica, y sus dimensiones dependen de varios segmentos del esqueleto, como el cefálico, raquídeo (altura de la columna), pelviano y de los miembros inferiores, cada uno contribuye a la estatura del individuo en consideración a la edad, sexo, raza, condiciones socioeconómicas y psicosociales, y finalmente las tendencias históricas (Rodriguez, 1994). Para Krishan et al. (2010), la estimación de la estatura es un parámetro importante en la identificación de los restos óseos durante los exámenes forenses.

En los últimos años se han propuesto varios métodos para la estimación de la estatura. Entre éstos, está la reconstrucción anatómica y la ecuación de regresión con base en los huesos largos. El primer método, es preferible porque tiene en cuenta la altura total del esqueleto y por lo tanto proporciona estimaciones más precisas, pero no se puede aplicar a los restos incompletos (Vercellotti et al., 2009).

\footnotetext{
* Cirujano Dentista, práctica privada, Chile.

* Universidad Autónoma de Chile, Talca, Chile.

${ }^{* * *}$ Facultad de Medicina, Universidad Diego Portales, Santiago, Chile.
} 
Y el segundo, es una relación métrica de la longitud del hueso respecto a la longitud total del cuerpo. Sin embargo, las distintas poblaciones requieren ecuaciones diferentes debido a que poseen distintas proporciones corporales. Para evitar aquello, se utiliza una estatura femoral media (proporción de las distintas ecuaciones, que proporcionará una estimación adecuada de la estatura con un margen de error de 5 cm (Bahn \& Renfrew, 1993).

Los métodos más confiables para estimar la estatura cuando solo contamos con restos óseos, exigen la conservación de estos huesos largos, pero muy a menudo esto no es posible, por lo que es necesario el desarrollo de métodos alternativos (Cordeiro et al.).

Frente a este escenario, se recurre a la Odontología Forense, disciplina que aplica los conocimientos estomatológicos para el correcto examen, manejo, valoración y presentación de las pruebas buco-dentales en interés del derecho Laboral, Civil y Penal (Silva, 1997; Valdés et al., 2010), siendo relevante utilizar los elementos dentales disponibles, ya que el tejido dental puede resistir condiciones extremas de degradación, como la exposición a altas temperaturas, la humedad y la presión excesiva. El alto contenido mineral de los tejidos dentales, especialmente del esmalte, es responsable de su dureza y resistencia, por lo que permite la identificación de los cuerpos humanos (Ramenzoni \& Line, 2006).

En 1920, Ubaldo Carrea elaboró un método matemático que permite calcular la estatura del sujeto a partir de las dimensiones dentales (Carrea, 1920; Carrea, 1939). Carrea, parte de los diámetros mesiodistales de un incisivo central, lateral y canino inferiores, cuya suma en milímetros constituye un arco de circunferencia que abarca a estos tres dientes. La cuerda de este arco y la denomina Radio-cuerda inferior son las medidas fundamentales del llamado diagrama dentario propuesto por Carrea (Briñon, 1984). Este método ha sido evaluado por distintos autores sin resultados concluyentes sobre la utilidad del método, no obstante su uso éxito en procedimientos de interés púbico como la identificación de Josef Mengele en Brasil (Silva, 1990; Sampaio, 1995; Cavalcanti et al., 2007). Este método también fue evaluado en maxilar con resultados negativos (Lima et al., 2008; Lima, 2011), reportándose para una muestra de población chilena un $54 \%$ de acierto (Gajardo et al., 2011). No obstante el bajo rendimiento en el maxilar, es necesario desarrollar métodos que permitan estimar la estatura de un sujeto, a partir de dimensiones dentales individuales y en este arco dentario, en especial considerando que la mandíbula se desprende del cráneo y muchas veces se pierde. Adicionalmente no existen estudios que evalúen estos métodos en niños, no obstante la relativa estabilidad que presentan las dimensiones de los elementos dentales una vez erupcionados.

Con estos antecedentes, el propósito de este estudio es evaluar el método de Carrea en dentición temporal y proponer, mediante estadística multivariada, modelos matemáticos para la estimación de la estatura en niños a partir de las dimensiones de piezas dentarias temporales

\section{MATERIAL Y MÉTODO}

Participaron 42 pacientes pediátricos de entre 36 y 84 meses de edad, de ambos sexos, que acudieron a evaluación odontológica, los cuales junto a sus padres o cuidadores fueron informados de la naturaleza del estudio y entregaron una constancia escrita de su adhesión.

Se incorporaron pacientes con sus incisivos centrales, laterales y caninos temporales superiores erupcionados. No se consideraron pacientes con agenesias dentarias o piezas supernumerarias, con destrucción coronaria extensa o fracturas de las piezas a evaluar. Una vez seleccionada la muestra, a cada paciente se le determinó su estatura real.

Posteriormente utilizando un caliper se midieron los diámetros mesiodistales del incisivo central, lateral y canino temporal superior, cuya suma en milímetros constituyó el arco referido por Carrea, del mismo modo se estableció el radio-cuerda, determinado por una recta que entre el margen mesial del incisivo central y el margen distal del canino respectivamente (Fórmula), utilizando el método de remedición se calculó la confiabilidad de las mediciones.

$\frac{\text { Estatura máxima: } \operatorname{arco}(\mathrm{mm}) \times 6 \times 3,1416 \times 10}{2}$

$\frac{\text { Estatura mínima: radio-cuerda }(\mathrm{mm}) \times 6 \times 3,1416 \times 10}{2}$

Fórmula propuesta por Correa para la determinación de la estatura 
Obtenidas ambas mediciones se aplicó el modelo matemático propuesto por Carrea (Fig. 2) y la estimación se comparó con la estatura real.

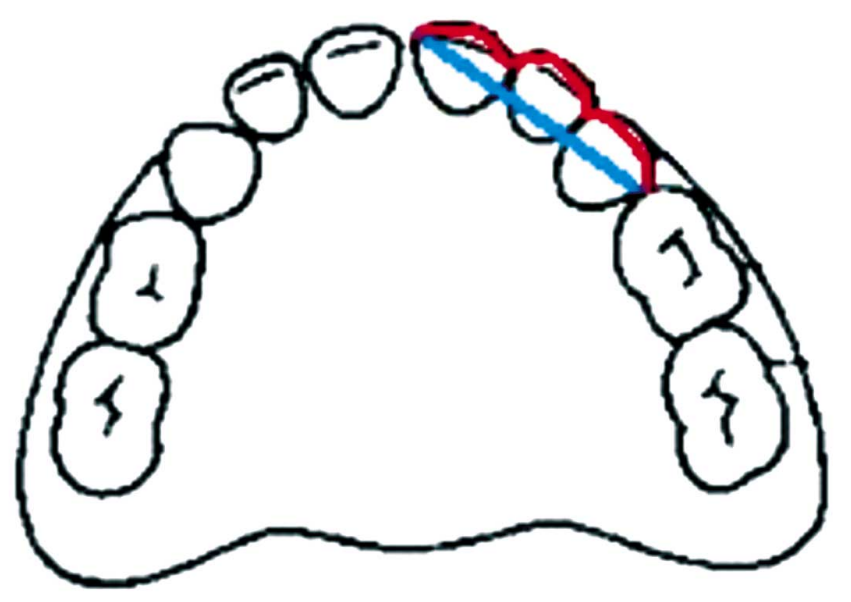

Fig. 2. Esquema del arco (Rojo) y radio-cuerda (azul) en el maxilar

\section{RESULTADOS}

La estatura media fue de $115 \mathrm{~cm}$ (DE 0,09). Se pudo observar que la estatura mínima fue de $96 \mathrm{~cm}$ y la máxima de $128 \mathrm{~cm}$. La media del arco fue de 16,98 (DE 0,81) en el lado derecho y $17,01(0,86)$ en el lado izquierdo, mientras que el radio cuerda derecho fue $18,46(\mathrm{DE} 0,89)$ y el izquierdo 18,58 (DE 1,1). El coeficiente correlación interclase fue de 0,92.

El método de Carrea resultó negativo en todos los casos por lo que se realizó una regresión lineal multivariada para el cálculo de estatura a partir de las dimensiones de la dentición temporal.

Regresión lineal multivariada en maxilar. Se realizó la regresión lineal de los datos arco maxilar y radiocuerda maxilar ambos del lado derecho.

Al determinar la ecuación de regresión se observó que el coeficiente de determinación era demasiado bajo $\left(r^{2}=0,01\right)$, por lo que se decidió excluir del análisis los casos extremos (niños con edades de 36,48 y 84 meses). Con esto el grupo de análisis quedó constituido por 33 individuos de 60 y 72 meses.

Se realizó la regresión lineal multivariada con los datos arco maxilar derecho, radio-cuerda maxilar derecho como variables independientes y la estatura en $\mathrm{mm}$ como variable dependiente.

La ecuación resultante fue:

Estatura $=1358,798+($ Arco $x-8,577)+($ Radio-cuerda $x-1,914)$. El valor $r^{2}$ fue de 0,021 .

Para evaluar la utilidad práctica del método, se realizó la validación cruzada de los datos, con ello se determinó que la fórmula estatura $\pm 5 \mathrm{~cm}$ permitía estimar la estatura en un $57,5 \%$ de los casos, mientras que la fórmula estatura $\pm 10 \mathrm{~cm}$ permitió estimar la estatura en un $97 \%$ de los casos.

\section{DISCUSIÓN}

La antropología forense emplea métodos reconstructivos para establecer la identidad de cadáveres deteriorados o fragmentos de éstos a través de la determinación de la edad, estatura, sexo y raza, elementos básicos y principales de la identificación médico legal.

A través de los años, la determinación de la estatura de restos humanos para fines de identificación ha sido confiada casi exclusivamente a los métodos basados en la medición de huesos largos. Sin embargo, existen casos en los que estas piezas óseas no se encuentran disponibles y, por lo tanto, esos métodos no son aplicables; es en estas situaciones en las que es de utilidad un método que estime la estatura a partir de mediciones dentarias, como el elaborado por Ubaldo Carrea, quien se basó en la Odontometría, señalando que los dientes son proporcionales al conjunto de la arcada, la cabeza y el sujeto en general.

En nuestro estudio determinamos que el método de Carrea no es aplicable a la dentición temporal maxilar, lo que concuerda con los bajos resultados obtenidos por Gajardo et al. y por Lima et al. La ineficiencia de este método puede deberse a, según lo propuesto por Carrea, que las proporciones faciales y de estatura se basan siempre en la mandíbula, a partir del triangulo Mandibular de Bonwill (Carrea, 1920). La proporción de 6 radios cuerda determinan el lado dicho triángulo y 7 radios cuerda determinan el triangulo externo, que extrapola las dimensiones faciales y de las que se desprende la fórmula para la determinación de la estatura en adultos. 
Una mayor exactitud encontramos al aplicar una regresión lineal multivariada con un $57,5 \%$ de exactitud al considerar un rango de $\pm 5 \mathrm{~cm}$ y un $97 \%$ de exactitud al considerar un rango sobre la fórmula de $\pm 10 \mathrm{~cm}$.

El modelo matemático descrito puede ser utilizado en diversas situaciones, ya que el maxi- lar debido a su posición y al estar unido al cráneo presenta un menor índice de fractura en comparación con la mandíbula en muertes traumáticas, lo que facilitaría su uso con todos sus componentes dentarios, sin embargo este debe ser complementado con otros métodos para la estimación de la estatura.

GARRIDO, Y.; ZAVANDO, D. \& SUAZO, G. I. Estimation of stature from dimensions of the deciduous dentition. Int. J. Odontostomat., 6(2):139-143, 2012.

ABSTRACT: The estimation of stature from bone or tooth segments is a complex procedure with questionable validity, especially when dealing with human remains of subadults. The aim of this study was to evaluate the Carrea method for determining stature from the dimensions of deciduous maxillary teeth and in a second step to propose a suitable mathematical method for estimating stature. We determined the arch and radio-cord maxillary in 42 patients between 36 and 84 months and applied the Carrea method, comparing estimated with actual stature. Subsequently a multivariate linear regression with stature as dependent variable and the the arch and radio-cord maxillary as independent variable. Carrea method was negative in all cases. We determined a regression equation that allowed the correct estimation of stature in $57.5 \%$ of cases with $\pm 5 \mathrm{~cm}$ and in $97 \%$ with $\pm 10 \mathrm{~cm}$, which is a handy utility for estimating the stature that is complemented with other methods.

KEY WORDS: stature, index of Carrea, forensic dentistry.

\section{REFERENCIAS BIBLIOGRÁFICAS}

Bahn, P. \& Renfrew, C. Arqueología: teorías, métodos y práctica. Madrid, Ediciones Akal, 1993. p.395.

Borborema, M.; Vanrell, J. \& Queluz, D. Height estimation trough limbs long bones and pelvis bones measurement. Odonto, 18(36):113-25, 2010.

Briñón, E. N. Odontología legal y práctica forense. Buenos Aires, Purizón, 1984.

Carrea, J. U. Ensayos Odontométricos. Tesis. Buenos Aires, Universidad Nacional de Buenos Aires, 1920.

Carrea, J. U. Talla individual humana en función al radio cuerda. Ortodoncia, 6:225-7, 1939.

Cavalcanti, A. L.; Porto, D. E.; Maia, A. M. A. \& Melo, T. R. N. B. Estimativa da estatura utilizando a análise dentária: estudo comparativo entre o método de Carrea e o método modificado. Rev. Odontol. UNESP, 36:335-9, 2007.

Cordeiro, C.; Muñoz-Barús, J. I.; Wasterlain, S.; Cunha, E. \& Vieira, D. N. Predicting adult stature from metatarsal length in a Portuguese population. Forensic Sci. Int., 193(1-3):131.e1-4, 2009.
Gajardo, P.; Gajardo, M.; Torres, S.; Zavando, D. \& Suazo, G. I. Determinación de la estatura a partir del arco y radio-cuerda maxilar. Int. J. Odontostomat., 5(3):267-9, 2011.

Krishan, K.; Kanchan, T. \& DiMaggio, J. A. A study of limb asymmetry and its effect on estimation of stature in forensic case work. Forensic Sci. Int., 200(1-3):181.e1-5, 2010.

Lima, L. N. C. Validação do índice de Carrea por meio de elementos dentais superiores para a estimativa da estatura humana. Dissertação Mestrado. Universidade Estadual de Campinas, Fac. de Odontologia de Piracicaba, 2011.

Lima, L. N. C; Neves, G. L. S \& Rabello, P. M. Carrea's index in dental students at the Federal University of Paraíba. Braz. J. Oral Sci., 7(2):1673-7, 2008.

Mantilla, J.; Cardenas, N. \& Jácome, J. Estimation of Height from Measurements of the Tibia in Colombian Population. Int. J. Morphol., 27(2):3059, 2009.

Ramenzoni, L. L. \& Line, S. R. Automated biometrics- 
based personal identification of the HunterSchreger bands of dental enamel. Proc. Biol. Sci., 273(1590):1155-8, 2006.

Rodriguez, J. V. Reconstrucción de la estatura. En: Introducción a la Antropología Forense: Análisis e identificación de restos óseos humanos. Bogotá, Universidad Nacional de Colombia Santafé de Bogotá, 1994.

Sampaio, C. M. A. Avaliação do índice de Carrea comparado ao índice cefalométrico de Retzius e índice facial. Dissertação mestrado. Universidade Estadual de Campinas, Faculdade de Odontologia de Piracicaba, 1995.

Silva, M. Compêndio de Odontologia Legal. São Paulo, Medsi, 1997.

Silva, M. Estimativa da estatura do indivíduo com a utilização de um grupo de dentes da mandíbula. Rev. Paul. Odontol., 12:18-28, 1990.

Suazo, G. I. C; Russo, P. P.; Zavando, M. D. A. \& Smith, R. L. Sexual dimorphism in the foramen magnum dimensions. Int. J. Morphol., 27(1):21-3, 2009a.

Suazo, G. I.; Zavando, M. D. \& Smith, R. L. Performance evaluation as a diagnostic test for traditional methods for forensic identification of sex. Int. J. Morphol., 27:381-6, 2009b.

Suazo, G. I. C; Zavando, M. D. A. \& Smith, R. L. Evaluating accuracy and precision in morphologic traits for sexual dimorphism in malnutrition human skull: a comparative study. Int. J. Morphol., 26(4):876-83, 2008.

Valdés, L.; Beltrán, B.; De la Garza-Ramos, M. \& Torres, A. Identificación humana basado en técnicas odontológicas forenses; propuesta de base de datos. Revista Oral, 11(S2):18-20, 2010.

Vercellotti, G.; Agnew, A. M.; Justus, H. M. \& Sciulli, PW. Stature estimation in an early medieval (XIXII c.) Polish population: testing the accuracy of regression equations in a bioarcheological sample. Am. J. Phys. Anthropol., 140(1):135-42, 2009.
Dirección para correspondencia:

Prof. Dr. Iván Suazo G.

Departamento de Morfofunción

Facultad de Medicina

Universidad Diego Portales

Email: ivan.suazo@udp.cl

Recibido : 15-03-2012

Aceptado: 26-05-2012 